\title{
Regulatory Role of ACTH on Aldosterone in Aldosterone-Producing Adenoma
}

\author{
Takuhiro Sonoyama', Masakatsu Sone ${ }^{2 *}$ \\ 'Medical Innovation Center, Kyoto University Graduate School of Medicine, 53 Shogoin Kawahara-cho, Sakyo-ku, Kyoto 606-8507, Japan \\ 2Department of Diabetes, Endocrinology and Nutrition, Kyoto University Graduate School of Medicine, 54 Shogoin Kawahara-cho, Sakyo-ku, Kyoto 606-8507, \\ Japan
}

Article Info

\section{Article Notes}

Received: March 07, 2017

Accepted: May 08, 2017

\section{${ }^{*}$ Correspondence:}

Masakatsu Sone, MD, PhD; Department of Diabetes,

Endocrinology and Nutrition, Kyoto University Graduate

School of Medicine, 54 Shogoin Kawahara-cho, Sakyo-ku,

Kyoto 606-8507, Japan, Tel: +81-75-751-3170; Fax: +81-75-

771-9452, E-mail: sonemasa@kuhp.kyoto-u.ac.jp

(c) 2017 Masakatsu Sone. Cavalieri. This article is distributed under the terms of the Creative Commons Attribution 4.0

International License.

\section{Keywords}

Primary hyperaldosteronism

Aldosterone

Hypertension

Adrenal gland

Adrenocorticotropic hormone

Aldosterone-producing adenoma

\section{ABSTRACT}

Primary aldosteronism (PA), one of the most frequent causes of secondary hypertension, is mainly composed of two major subtypes: aldosteroneproducing adenoma (APA) and bilateral hyperaldosteronism (BHA). In APA, ACTH plays a dominant role in the regulation of circulating aldosterone level, while in physiological condition and BHA angiotensin II has a stronger effect. This pronounced regulation of aldosterone by ACTH in APA causes a differential response of aldosterone to either ACTH stimulation or ACTH suppression in APA from that in physiological condition and $\mathrm{BHA}$, and therefore, $\mathrm{ACTH}$ stimulation test can be informative in differentiating APA among patients with PA and essential hypertension. Histologic studies have suggested a possibility that the origin of APA especially consisting of clear, lipid rich cells could be zona fasticulata, rather than zona glomerulosa. Recent studies have been focusing on molecular classification among APA, which could lead to a better understanding of ACTH responsiveness in APA.

\section{Abbreviations}

ACTH: adrenocorticotropic hormone,

AII: angiotensin II

APA: aldosterone-producing adenoma,

AVS: adrenal venous sampling,

BHA: bilateral hyperaldosteronism,

MC2R: melanocortin 2 receptor,

PA: primary aldosteronism,

PAC: plasma aldosterone concentration,

StAR: steroidogenic acute regulatory protein,

ZF: zona fasciculate,

ZG: zona glomerulosa,

Primary aldosteronism (PA) is a pathological condition where aldosterone is over-produced from the adrenal gland either unilaterally or bilaterally, independent of the renin-angiotensin system which in normal condition is in charge of regulating aldosterone production ${ }^{1}$. PA is one of the most prevalent forms of secondary hypertension, and is considered to account for more than $5 \%$ of all hypertensives ${ }^{2-4}$. As a result of excess aldosterone, PA is clinically characterized by hypertension, high aldosterone/ 
renin ratio, and sometimes hypokalemia ${ }^{1}$. Detection of PA cases among hypertensive patients is essential because patients with PA have been known to be at risk of poorer cardiovascular and renal outcomes compared to those with essential hypertension even after adjustment for the blood pressure $^{5,6}$.

Aldosterone-producing adenoma (APA) is a form of $\mathrm{PA}$ in which excess aldosterone is secreted from a benign tumor in the adrenal gland ${ }^{1,7}$. In this Mini-review, we focus on the role of adrenocorticotropic hormone (ACTH) on blood aldosterone level in cases of APA and compare it to that in cases of other forms of PA.

Aldosterone-producing adenoma (APA) and bilateral hyperaldosteronism (BHA) are two major subtypes of primary aldosteronism (PA)

PA has two major subtypes; aldosterone-producing adenoma (APA) and bilateral hyperaldosteronism $(\mathrm{BHA})^{1,7,8}$. In APA, aldosterone is produced and secreted from a functional tumour usually occurring in the adrenal glands ${ }^{1,7,8}$. Aside from rare cases of APA which have functional tumours in the both adrenal glands, aldosterone production in APA is from one side of the adrenal glands on which the functional tumour resides. In contrast, BHA is a pathological condition where hyperplasia of both sides of zona glomerulosa of the adrenal gland occurs and autonomously overproduced aldosterone is secreted from both sides of the adrenal glands ${ }^{1,7,8}$. As factors causing hyperplasia in BHA are unknown, BHA is also known as idiopathic hyperaldosteronism (IHA). The differential diagnosis between BHA and APA is important since the treatment strategies for these two pathogenic conditions are different: APA can be surgically excised and cured, whereas BHA is usually treated medically with aldosterone receptor blockers ${ }^{7}$.

The currently accepted gold standard for the differentiation between APA and BHA is adrenal venous sampling (AVS) ${ }^{7-9}$. In AVS, blood samples are collected from left adrenal vein, right adrenal vein, and vena cava through a catheter usually inserted via the femoral vein, and the ratio of aldosterone over cortisol level in the samples is used for evaluation for the localization of the disease ${ }^{9}$. For those patients who seek surgical cure of APA, it is essential to perform AVS prior to surgery in order to confirm the diagnosis $^{7}$. However, given the high experty required to perform $\mathrm{AVS}^{9,10}$, and the consequent, limited capacity of AVS, it should be reserved for those highly suspicious of APA. Therefore, an easier and non-invasive diagnostic test has been looked for to select cases highly likely to have APA.

\section{Differential regulation of blood aldosterone level} in APA and BHA

Under physiological conditions, the blood aldosterone level is regulated mainly by angiotensin II (AII) and serum potassium level, and, to a lesser extent, by adrenocorticotropic hormone (ACTH $)^{1}$. ACTH is a pituitary hormone consisting of 39 amino acids and binds its specific receptor, melanocortine 2 receptor ${ }^{11}$, expressed by the adrenocortical cells. Upon its binding to MC2R, ACTH acutely activates intracellular cAMP-protein kinase A pathway, thereby increasing the expression of steroidogenic acute regulatory protein $(\mathrm{StAR})^{12}$, which regulates the production of steroids ${ }^{12}$. In zona glomerulosa (ZG) of the adrenal cortex which is the major source of circulating aldosterone under physiological condition, upregulated StAR results in the increase in the production of aldosterone ${ }^{12}$.

Since 1970s, there have been reports which show there is a difference in the regulation of aldosterone between APA and $\mathrm{BHA}^{13-17}$. In BHA, the regulation of aldosterone by AII has been considered dominant rather than that by $\mathrm{ACTH}^{13}$, as in the case of normal subjects. However, in APA, ACTH is known to exert a stronger role to stimulate aldosterone than in the case of BHA and normal subjects ${ }^{14,15}$. Moreover, in APA, regulation of aldosterone by ACTH has been considered to be dominant over that by $\mathrm{AII}^{16,17}$, although some report that AII-responsive types of APA (AII-R APA) account for a considerable percentage of APAs ${ }^{18,19}$. The mechanism of the stronger effect of ACTH on aldosterone level in APA has yet to be fully unveiled: a paper showed a higher expression level of ACTH receptor in $\mathrm{APAs}^{20}$, but the causality has not been addressed.

The pronounced regulation of aldosterone by ACTH in cases of APA prompted us to think of ACTH stimulation test as a possible diagnostic test of APA and to examine the diagnostic efficiency of this test in a larger number of patients and in a more systematic way than earlier studies. In 2011, we reported that ACTH stimulation test after dexamethasone administration is a useful procedure to detect APA among those with suspected PA, with a high diagnostic accuracy ${ }^{21}$. We adopted dexamethasone administration the night before ACTH stimulation test in order to suppress endogenous ACTH and to minimize its effect $^{21}$.

After our report, another group has performed a similar study analyzing the diagnostic capacity of ACTH stimulation test under dexamethasone suppression to diagnose APA, with the result consistent with ours in that the test is useful for detecting unilateral $\mathrm{PA}^{22}$. There have also been studies that tested the efficiency of ACTH stimulation test without dexamethasone suppression, but they were not so successful in differentiating the two major subtypes in PA patients ${ }^{23,24}$, suggesting the importance of dexamethasone administration. As shown in a previous study, dexamethasone administration can suppress the fluctuation of aldosterone level caused by endogenous 
$\mathrm{ACTH}^{25}$, and may help to line up the baseline aldosterone level before ACTH administration.

We also thought of the possibility that there could be differences in circadian change and/or the change after dexamethasone administration of plasma aldosterone between APA and BHA. Hence, we examined circadian change in plasma aldosterone concentration (PAC), and changes in PAC after dexamethasone administration in patients with suspected PA, in order to evaluate the effect of endogenous ACTH on aldosterone secretion ${ }^{26}$. As expected, the change of blood aldosterone level in APA patients after dexamethasone administration was greater than those in IHA and non-PA patients ${ }^{26}$, indicating that aldosterone secretion in APA patients is more strongly dependent on endogenous ACTH than in BHA and non$\mathrm{PA}^{26}$. In patients with BHA and non-PA patients, blood aldosterone levels showed circadian decrease towards the night but did not correlate with ACTH levels. This indicates that factors other than ACTH may cause circadian changes in aldosterone secretion in those patients. A report by Doi et al. demonstrated that mice lacking core clock genes, cryptochrome- 1 and cryptochrome- 2 showed the phenotype of $\mathrm{BHA}^{27}$, and this may suggest that clock genes are involved in the regulation of aldosterone production from the adrenal gland.

These two tests, ACTH stimulation test and dexamethasone suppression test, can be helpful in detecting cases highly likely to have APA, although they cannot be confirmative for the final diagnosis of APA because of the considerable number of cases of APA and BHA in which aldosterone does not respond as expected. However, they can be informative in choosing cases to be prioritised for AVS.

\section{Molecular stratification of APA can predict ACTH- responsiveness}

From prior years, it has been known that there are several different cell types found in $\mathrm{APA}^{24}$. One type of the cells have little lipid storage in cytoplasm and morphologically resemble the cells in zona glomerulosa (ZG) of the adrenal gland ${ }^{28}$, which is the site of physiological aldosterone production under the regulation of $\mathrm{AII}^{28}$. Another type of cells have large amounts of intracellular lipid and are similar to zona fasciculata (ZF) cells ${ }^{28}$ (ZF cells are responsible for producing and secreting cortisol, rather than aldosterone, in normal condition and express higher level of ACTH receptor than ZG cells). Also, a hybrid of the two types of cells above has been reported ${ }^{28}$.

There have been reports regarding the cell types and differential regulation in aldosterone production ${ }^{29}$. One report showed that APAs consisting of ZG-like cells are more responsive to angiotensin II than those consisting of ZF-like cells ${ }^{29}$. This may indicate different origins of APAs consisting of these different cell types, because APA with clear, ZF-like, cells might be considered to develop from $\mathrm{ZF}$, rather than $\mathrm{ZG}$, given the morphological similarity and unresponsiveness to angiotensin II. Meanwhile, another paper demonstrated that angiotensin II responsive APA, mainly consisting of ZG-like cells, is as responsive to ACTH as angiotensin II unresponsive $\mathrm{APA}^{19}$.

Recently, researchers have been trying to understand the pathophysiology of APA on a molecular basis. Since the report by Choi et al in 2011 which identified somatic mutations of KCNJ5, a potassium voltage-gated channel, in some cases of $\mathrm{APAs}^{30}$, there have been numerous reports regarding somatic mutations in APAs and, in addition to KCNJ5, mutations of calcium voltage-gated channel subunit alpha 1D (CACNA1D; a calcium voltage-gated channel) ${ }^{31}$, ATP1A1 and ATP2B3 (ATPases) ${ }^{32}$ have been identified in APAs so far. Phenotypic differences between those APAs with or without such mutations have also been reported, and nowadays, APAs have come to be characterised according to the somatic mutations they harbor. According to a report by Azizan in 2012, APAs with KCNJ5 mutation are more likely to consist of ZF-like, clear cells than those without KCNJ5 mutation ${ }^{33}$. A more recent study by Kitamoto et al. showed that APAs with somatic mutation of CACNA1D consisted mainly of ZF-like clear cells and blood aldosterone levels in these cases were more responsive to ACTH than those without the mutation ${ }^{34}$. Such molecular classification of APAs may provide a clearer image of the origin and the pathophysiological mechanism of APA in the near future. However, to identify mutations, samples of the APAs are inevitably required and, because the samples can only be obtained after surgical removal, the molecular classification of APA is only informative retrospectively, with limited capacity for prospective diagnosis in a clinical setup.

In conclusion, blood aldosterone level in APAs is more sensitive to ACTH than those in BHA and normal subjects, which is informative in the selection of those patients highly likely to have APA prior to AVS. Although the precise mechanism underlying this has yet to be unraveled, molecular classification of APAs depending on somatic mutations they have can be informative in understanding the pathophysiology of APAs.

\section{References}

1. Young WF, Eds Melmed S, Polonsky KS, et al. Endocrine Hypertension In Williams Textbook of Endocrinology edn 12 ch 6. Main Saunders. 2011; pp 545-577.

2. Rossi GP, Bernini G, Caliumi C, et al. For the PAPY Study Investigators A prospective study of the prevalence of primary aldosteronism in 1125 hypertensive patients. J Am Coll Cardiol. 2006 Dec 5; 48(11): 2293-300.

3. Fardella C, Mosso L, Gómez Sánchez C, et al. Primary aldosteronism in essential hypertensives: prevalence, biochemical profile, and molecular biology. J Clin Endocrinol Metab. 2000 May; 85(5): 1863-7. 
4. Mulatero P, Dluhy R, Giacchetti G, et al. Diagnosis of primary aldosteronism: from screening to subtype differentiation. Trends Endocrinol Metab. 2005 Apr; 16(3): 114-9.

5. Milliez P, Girerd X, Plouin P, et al. Evidence for an increased rate of cardiovascular events in patients with primary aldosteronism. J Am Coll Cardiol. 2005 Apr 19; 45(8): 1243-8.

6. Sechi LA, Novello M, Lapenna R, et al. Long-term renal outcomes in patients with primary aldosteronism. JAMA. 2006 Jun 14; 295(22): 2638-45.

7. Funder JW, Carey RM, Mantero F, et al. The Management of Primary Aldosteronism Case Detection Diagnosis and Treatment: An Endocrine Society Clinical Practice Guideline. J Clin Endocrinol Metab. 2016 May; 101(5): 1889-916.

8. Young WF. Primary aldosteronism: renaissance of a syndrome. Clin Endocrinol Oxf. 2007 May; 66(5): 607-18.

9. Rossi GP, Auchus RJ, Brown M, et al. An expert consensus statement on use of adrenal vein sampling for the subtyping of primary aldosteronism. Hypertension. 2014 Jan; 63(1): 151-60.

10. Young WF, Stanson AW. What are the keys to successful adrenal venous sampling (AVS) in patients with primary aldosteronism. Clin Endocrinol Oxf. 2009 Jan; 70(1): 14-7.

11. Mountjoy KG, Robbins LS, Mortrud MT, et al. The cloning of a family of genes that encode the melanocortin receptors. Science. 1992; 257(5074): 1248-51.

12. Hattangady NG, Olala LO, Bollag WB, et al. Acute and chronic regulation of aldosterone production. Mol Cell Endocrinol. 2012 Mar; 350(2): 151-62.

13. Wisgerhof M, Brown RD, Hogan MJ, et al. The plasma aldosterone response to angiotensin II infusion in aldosterone-producing adenoma and idiopathic hyperaldosteronism. J Clin Endocrinol Metab. 1981 Feb; 52(2): 195-8.

14. Mantero F, Gion M, Armanini D, et al. Aldosterone regulation in primary aldosteronism: differences between adenoma and bilateral hyperplasia. Clin Sci Mol Med Suppl. 1976 Dec; 3: 329s-332s.

15. Kem DC, Weinberger MH, Higgins JR, et al. Plasma aldosterone response to ACTH in primary aldosteronism and in patients with low renin hypertension. J Clin Endocrinol Metab. 1978 Apr; 46(4): 552-60.

16. Saruta T, Okuno T, Eguchi T, et al. Responses of aldosterone-producing adenomas to ACTH and angiotensins. Acta Endocrinol Copenh. 1979 Dec; 92(4): 702-9.

17. Schambelan M, Brust NL, Chang BC, et al. Circadian rhythm and effect of posture on plasma aldosterone concentration in primary aldosteronism. J Clin Endocrinol Metab. 1976 Jul; 43(1): 115-31.

18. Tunny TJ, Klemm SA, Stowasser M, et al. Angiotensin-responsive aldosterone-producing adenomas: postoperative disappearance of aldosterone response to angiotensin. Clin Exp Pharmacol Physiol. 1993 May; 20(5): 306-9.

19. Stowasser M, Klemm SA, Tunny TJ, et al. Plasma aldosterone response to ACTH in subtypes of primary aldosteronism. Clin Exp Pharmacol Physiol. 1995 Jun-Jul; 22(6-7): 460-2.

20. Reincke M, Beuschlein F, Latronico AC, et al. Expression of adrenocorticotrophic hormone receptor mRNA in human adrenocortical neoplasms: correlation with P450scc expression. Clin Endocrinol Oxf. 1997 May; 46(5): 619-26.

21. Sonoyama T, Sone M, Miyashita $K$, et al. Significance of adrenocorticotropin stimulation test in the diagnosis of an aldosterone-producing adenoma. J Clin Endocrinol Metab. 2011 Sep; 96(9): 2771-8.

22. Jiang Y, Zhang C, Wang W, et al. Diagnostic value of ACTH stimulation test in determining the subtypes of primary aldosteronism. J Clin Endocrinol Metab. 2015 May; 100(5): 1837-44.

23. Umakoshi H, Xiaomei $\mathrm{Y}$, Ichijo T, et al. WAVES-J Study Group. Reassessment of the cosyntropin stimulation test in the confirmatory diagnosis and subtype classification of primary aldosteronism. Clin Endocrinol Oxf. 2017 Feb; 86(2): 170-6.

24. Terui K, Kageyama K, Nigawara T, et al. Evaluation of the (1-24) adrenocorticotropin stimulation test for the diagnosis of primary aldosteronism. J Renin Angiotensin Aldosterone Syst. 2016 Mar 23; 17(1): 1470320315625703.

25. Kem DC, Weinberger MH, Gomez Sanchez C, et al. The role of ACTH in the episodic release of aldosterone in patients with idiopathic adrenal hyperplasia, hypertension, and hyperaldosteronism. J Lab Clin Med. 1976 Aug; 88(2): 261-70.

26. Sonoyama T, Sone M, Tamura N, et al. Role of endogenous ACTH on circadian aldosterone rhythm in patients with primary aldosteronism. Endocr Connect. 2014 Dec; 3(4): 173-9.

27. Doi M, Takahashi Y, Komatsu R, et al. Salt-sensitive hypertension in circadian clock-deficient Cry-null mice involves dysregulated adrenal Hsd3b6. Nat Med. 2010 Jan; 16(1): 67-74.

28. Tsuchiyama H, Kawai K, Harada T, et al. Functional pathology of aldosterone-producing adenoma. Acta Pathol Jpn. 1980 Nov; 30(6): 967-76.

29. Tunny TJ, Gordon RD, Klemm SA, et al. Histological and biochemical distinctiveness of atypical aldosterone-producing adenomas responsive to upright posture and angiotensin. Clin Endocrinol Oxf. 1991 May; 34(5): 363-9.

30. Choi M, Scholl UI, Yue P, et al. K+ channel mutations in adrenal aldosterone-producing adenomas and hereditary hypertension. Science. 2011 Feb 11; 331(6018): 768-72.

31. Scholl UI, Goh G, Stölting G, et al. Somatic and germline CACNA1D calcium channel mutations in aldosterone-producing adenomas and primary aldosteronism. Nat Genet. 2013 Sep; 45(9): 1050-4.

32. Beuschlein F, Boulkroun S, Osswald A, et al. Somatic mutations in ATP1A1 and ATP2B3 lead to aldosterone-producing adenomas and secondary hypertension. Nat Genet. $2013 \mathrm{Apr}$; 45(4): 440-4, 444e1-2.

33. Azizan EA, Lam BY, Newhouse SJ, et al. Microarray qPCR and KCNJ5 sequencing of aldosterone-producing adenomas reveal differences in genotype and phenotype between zona glomerulosa- and zona fasciculata-like tumors. J Clin Endocrinol Metab. 2012 May; 97(5): E819-29.

34. Kitamoto T, Suematsu S, Yamazaki Y, et al. Clinical and Steroidogenic Characteristics of Aldosterone-Producing Adenomas With ATPase or CACNA1D Gene Mutations. J Clin Endocrinol Metab. 2016 Feb; 101(2): 494-503. 